\title{
LVIII. On the distribution of flow in a strained elastic solid
}

\section{Charles A. Carus-Wilson B.A. A.M.I.C.E.}

To cite this article: Charles A. Carus-Wilson B.A. A.M.I.C.E. (1890) LVIII. On the distribution of flow in a strained elastic solid, Philosophical Magazine Series 5, 29:181, 503-507, DOI: 10.1080/14786449008619975

To link to this article: http://dx.doi.org/10.1080/14786449008619975

曲 Published online: 08 May 2009.

Submit your article to this journal $\lceil\pi$

Џ Article views: 2

Q View related articles $\asymp$ 
The method of using the still is as follows:-The tap $\mathbf{E}$ is opened, $F$ is closed; a water-pump then exhausts the whole system, and the mercury to be acted on rises from the cistern $\mathrm{A}$. The cistern being large and shallow, only a slight change takes place in the height of the mercury in the bulb, when the level of the mercury in the cistern changes. While the pump is exhausting the ring of gas-jets is lit, and in about ten minutes, in the case of the still in our laboratory, the mercury fills the tube $\mathrm{D}$, any metal which comes over being caught in the bulb $G$. The tap $E$ is then closed and $F$ opened; the still then continues to work by virtue of the vacuum formed by its own mercury. It has been found necessary to place a gas-regulator on the pipe which supplies the jets, as the change of pressure in the gas-mains is considerable. An automatic arrangement, depending for its action upon the height of the mercury in the cistern, shuts off the gas when the surface of the mercury falls below a certain point. In using stills of this class the mercury before distillation should be carefully freed from moisture, as a minute quantity of water will often cause a fracture in the heated tube or bulb.

LVIII. On the Distribution of Flow in a Strained Elastic Solid. By Charles A. Carus-Wilson, B.A., A.M.I.C.E., Demonstrator in the Mechanical Laboratory at the Royal Indian Engineering College, Coopers Hill*.

TF a metal bar of uniform section throughout its length be subjected to uniform'longitudinal stress, the elements of the bar will become distorted ; if the strain should exceed the limit of elasticity the distortion will be partly permanent.

In consequence of the straining of the elements, every element will experience a displacement relative to three fixed axes in the bar; say $o x$ parallel to the length of the bar, oy parallel to one side, and $o z$ parallel to a second side, both $o z$ and $o y$ being at right angles to $o x$ : such displacement is generally spoken of as "flow."

It is clear that if the metal be homogeneous, the stress uniform, and the section at right angles to the axis everywhere the same, an element will experience a displacement at right angles to the planes $x o z, y o z$ proportional to its distance from these planes; since such displacement depends on the straining of the elements between it and these planes. Hence a bar with its sides originally parallel to $o x$ will remain so

* Communicated by the Physical Society : read May 2, 1890. $2 \mathrm{Q} 2$ 
after a certain amount of flow has taken place, and lines drawn on the sides respectively at right angles to and parallel to $o x$ will remain so, since the displacement parallel to $o x$ will be proportional to the distance from the plane yoz.

The question I propose to discuss is how to determine the distribution of flow when the section at right angles to $O x$ varies in any way as we pass along $o x$.

I will for simplicity consider a plate in which the thickness parallel to $o y$ is considerable compared with the thickness parallel to $o z$, and I will consider the displacements in the plane $x o y$.

We may take a plate in which there is a gradual reduction of section, in which there is what is commonly called a "shoulder," as in fig. 2.

It is required to determine the displacements in the plane of the paper-the plane $x \circ y$ : in other words, supposing the plate covered with a series of lines parallel to $o x$ and to $o y$ respectively, it is required to determine the curvature of these lines due to flow.

The distortion of the elements is caused by the shearingstrain induced in the bar when subjected to longitudinal stress. Since this shearing-strain is a maximum at $45^{8}$ to the axis $o \cdot t$ of the bar, it will be convenient to consider the bar divided

Fig. 1.

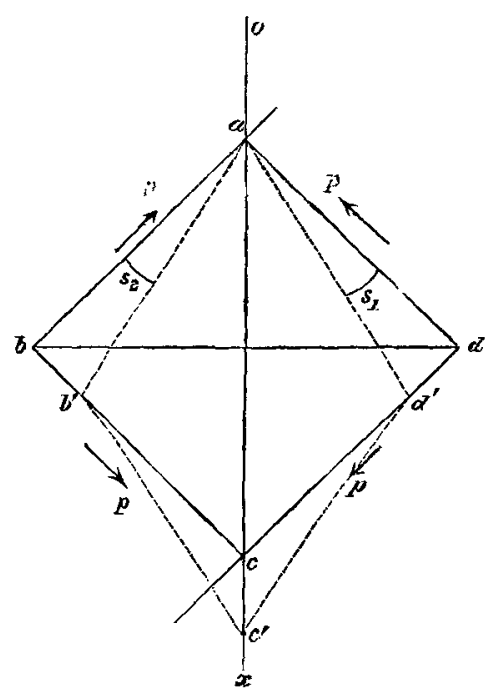

into a number of small elements with sides making angles of $45^{\circ}$ to the axis $o x$. Every such element as $a b c d$ (fig. 1) will 
be subject to a shearing-strain of amount $\frac{p}{n} ; i$.e. the angle $b a d$ will be diminished by an amount $\frac{p}{n}$, where $p$ is the intensity of the shearing-strain on the element, and $n$ is the coefficient of rigidity of the motal. The effect of this shearingstrain on the element may be described as a rotation of ad through an angle $s_{1}$, and of $a b$ through an angle $s_{2}$, where $s_{1}+s_{2}=\frac{p}{n} ; s_{1}$ and $s_{2}$ may be equal, when the displacement of $c$ will be wholly parallel to $o x$; or they may be unequal, when $c$ will have a displacement parallel to $o x$ and a resolved displacement parallel to $o y$, which will be + ve or - ve according as $s_{1}$ is less than or greater than $s_{2}$, and which will be a maximum if either $s_{1}$ or $s_{2}=0$.

The value of $s_{1}+s_{2}$, and the ratio of $s_{1}$ to $s_{2}$ for any element, appears to be determined by the following considerations. If a side $a b$ of the element be produced in both directions so as to cut the sides of the bar, there is seen to be a tendency of that part of the bar below this line to slide over that part which is above. Assuming the resulting shearing-strain to be uniform over the length $l_{1}$ of this oblique section, the shearing-strain at any element in this direction, $i . e . s_{1}$, will clearly be inversely proportional to $l_{1} ; s_{2}$ will also be inversely proportional to $l_{2}$, where $l_{2}$ is the length of an oblique section parallel to $a d$, and $s_{1}+s_{2}$ will be proportional to $\frac{l_{1}+l_{2}}{l_{1} l_{3}}$-say to $\frac{1}{\rho}$.

Suppose, now, we take a series of elements, $a_{1} b_{1} c_{1} d_{1}-$ $a_{2} b_{2} c_{2} d_{2}$ touching one another and lying across the bar. If we imagine the points $a_{1}, a_{2}, a_{3}$, \&c. fixed on a line parallel to $o y$, it is clear that the displacements of $c_{1}, c_{2}, c_{3}$, \&c. will be proportional to the sum of the shearing-strains in each element. If $s_{1}+s_{2}$ is the same for each element, and $s_{1}=s_{2}$, the points $c_{1}, c_{2}, c_{3}$, \&c. will remain on a line parallel to $o y$. If, however, $s_{1}+s_{2}$ becomes greater as we approach the middle, and less again towards the further side, the points $c_{1}, c_{2}, c_{3}$, \&c. will be found on a line concave upwards ; if $s_{1}+s_{2}$ diminishes towards the centre these points will lie on a line concave downwards. If the strains should exceed the limit of elasticity the line through $e_{1}, c_{2}, c_{3}$, \&c. will be permanently curved.

If the points $a_{1}, a_{2}, a_{3}, \ldots$, instead of being in one straight line have unequal downward displacements, the form of the curves obtained as above may be modified.

If, now, we consider a series of consecutive elements, $a_{1} b_{1} c_{1} d_{1}-a_{1} b_{2} c_{2} d_{2}$, lying in a line parallel to $o x$, if $s_{1}=s_{2}$ for 
each element the points $c_{1}, c_{2}, c_{3}$, \&c. will continue to lie on a line parallel to $o x$, since the displacement of each of these points, due to the distortion of the corresponding element, is parallel to $o x$. But if as we descend from the first element we find $s_{1}$ becoming greater than $s_{2}$, the points $c_{2}, c_{3}$, \&c. will have a displacement to the left which increases as we descend, so the line passing through $c_{1}, c_{2}, c_{3}$, \&c. will be bent; and if the shearing-strains become equal again on the lowest element, this line will be concave to the right.

It thus appears that any horizontal line will be curved concave downwards if the sum of $s_{1}+s_{2}$ is greater at the end of the line than in the centre, and vice vers $\hat{a}$, but that the line will remain straight if $s_{1}+s_{2}$ is constant along its length, and $s_{1}=s_{2}$.

Also that a vertical line will remain so if $s_{1}$ is everywhere equal to $s_{2}$; but if $s_{1}$ is $>s_{2}$, the line will be curved and concave towards the right, and vice versâ.

We can now proceed to determine the curvature of the lines in the case before us.

Commencing at the upper extremity of the bar, fig. 2, a line such as $a b$ will remain straight, because $\rho$ is everywhere constant, and $l_{1}=l_{2}$. The line cdef will be curved concave downwards, since from $c$ to $d$ and from $e$ to $f \rho$ is less than it is from $d$ to $e$ (where it is constant and $l_{1}=l_{2}$ ); de will therefore remain straight, and $c d$ and of curved as shown. The line $g h i$ will be concave downwards and curved along its entire length, since $\rho$ gets less as we move away from $h$ on both sides.

The line $j k l m n$ will remain straight from $j$ to $k$,

Fig. 2.

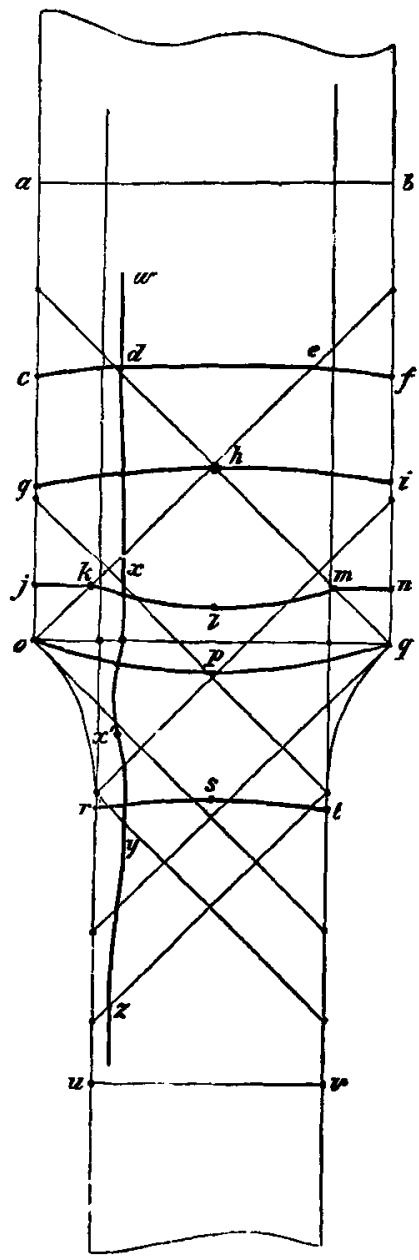
since $\rho$ is there constant and $l_{2}=l_{2}$; from $k$ to $l \rho$ becomes 
less and greater again from $l$ to $m, k l m$ is therefore concave upwards ; this line is straight from $m$ to $n$, since $\rho$ is constant and $l_{1}=l_{2}$.

The line $o p q$ is curved concave upwards throughout the entire length, since $\rho$ diminishes from $o$ to $p$ and increases from $p$ to $q$.

The line $r s t$ will be concave downwards, since $\rho$ diminishes from $r$ to $s$ and increases from $s$ to $t$.

The line $u v$ will remain straight, since $\rho$ is constant and $l_{1}=l_{2}$.

To turn now to the curvature of the vertical lines. If we consider one of these as $w x y z$, from $w$ to $d l_{1}=l_{2}$, at $x l_{2}$ is $<l_{1}$, while at $x^{\prime} l_{1}=l_{2}$ again, so that from $d$ to $x^{\prime}$ this line will be curved in convex towards ox. Again, at $y l_{2}$ is $\left\langle l_{1}\right.$, and at $z l_{1}=l_{2}$, therefore $x^{\prime} y z$ is also curved in convex towards $o x$.

It would appear then that the horizontal lines will have three distinct changes of curvature, while the vertical lines

Fig. 3.

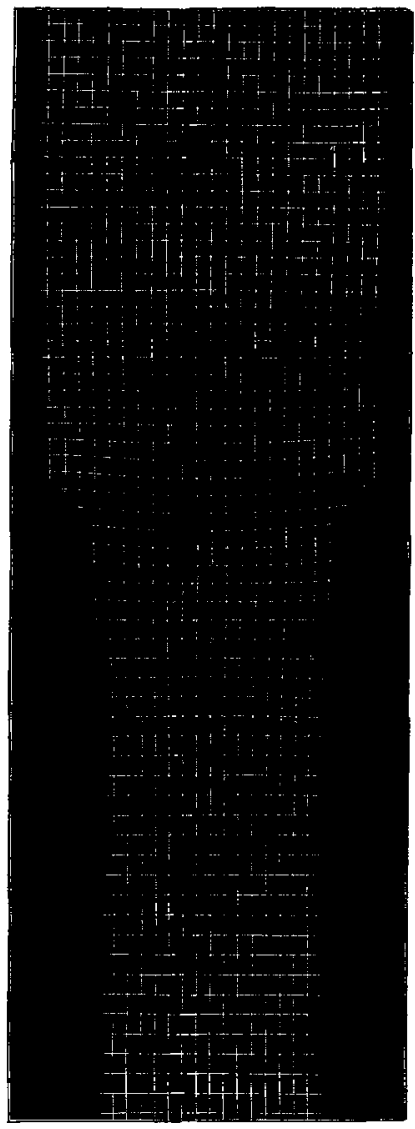
will be pinched in above and below the shoulder.

To test the accuracy of these conclusions, I prepared a copper bar as shown in the following way. The sides and faces were very carefully planed and polished, and on one face I inscribed a series of vertical and horizontal lines in a lathe. I then strained the bar so as to produce a considerable permanent set. An impression was then taken from the bar, which is reproduced in fig. $3^{*}$.

By placing the eye very nearly in the plane of the paper and looking along the lines, the curvatures can be seen to follow very closely those sketched on fig. 2 . I would specially draw attention to the partial curvature of such lines as $c d$ ef and $j k l m n$.

* The diagonal lines in fig. 3 were ruled on the impression to serve as lines of reference. 